DOI: $10.2478 /$ lpts-2020-0029

\title{
COMPUTER SIMULATION OF THE ELECTRIC TRANSPORT PROPERTIES OF THE FeSE MONOLAYER
}

\author{
D. Sergeyev1,2*, N. Zhanturina ${ }^{1}$, L. Myasnikova', \\ A.I. Popov ${ }^{3}$, A. Duisenova ${ }^{1}$, A. Istlyaup ${ }^{1}$ \\ ${ }^{1} \mathrm{~K}$. Zhubanov Aktobe Regional University, \\ 34 Alia Moldagulova Av., Aktobe, 030000, KAZAKHSTAN \\ $2 \mathrm{~T}$. Begeldinov Aktobe Aviation Institute, \\ 39 Alia Moldagulova Av., Aktobe, 030000, KAZAKHSTAN \\ ${ }^{3}$ Institute of Solid State Physics, University of Latvia, \\ 8 Kengaraga Str., Riga, LV-1063, LATVIA \\ *E-mail: serdau@mail.ru, nzhanturina@mail.ru, popov@latnet.Iv
}

The paper deals with the model research of electric transport characteristics of stressed and non-stressed FeSe monolayers. Transmission spectra, current-voltage characteristic (CVC) and differential conductivity spectra of two-dimensional FeSe nanostructure have been calculated within the framework of the density functional theory and non-equilibrium Green's functions (DFT + NEGF). It has been shown that the electrophysical properties depend on the geometry of the sample, the substrate, and the lattice constant. On CVC of non-stressed sample in the range from $-1.2 \mathrm{~V}$ to -1 and from $1.2 \mathrm{~V}$ to $1.4 \mathrm{~V}$, a region of negative differential resistance (NDR) has been observed. NDR is at both signs of the applied voltage due to the symmetry of the nanostructure. $d^{2} I / d V^{2}$ is used to determine the nature of the electron-phonon interaction and the features of quasiparticle tunnelling in stressed and non-stressed samples. The results obtained can be useful for calculating new elements of 2D nanoelectronics.

Keywords: Current-voltage characteristics, FeSe monolayer, transmission spectra, 2D-nanoelectronics. 
Currently, reducing the size of electronic components, which consist of the circuits of all modern chips, is becoming increasingly important [1]-[11]. For further miniaturization, it is necessary to develop new nanomaterials with controlled electrophysical properties [12]. With the discovery of graphene and other two-dimensional atomic crystals, the search for new 2D nanomaterials with unusual electrophysical properties began, as well as the development of various electronic devices based on it [13]-[15]. Various technological methods for synthesizing two-dimensional monoatomic crystals based on elements of the fourth group of the periodic table are already known: germanene [16], silicene [17], borophene [18], stanene [19], phosphorene [20], etc., as well as $2 \mathrm{D}$ crystals based on carbides, oxides, chlorides, nitrides, transition metal dichalcogenides, etc. [21]. Recently, intensive work has been carried out on the development of nanoelectronic devices based on 2D nanomaterials: field-effect transistors, sensors, power storage devices, solar cells, etc., so a new direction of nanoelectronics has appeared - 2D-nanoelectronics, the main idea of which is to implement elements of electronic technology using 2D-nanomaterials [22]. It opens up the possibility of producing completely new electronic devices with unusual characteristics.

At this moment, the properties of electronic nanomaterials are modelled by using various computer programs. Determining the transmission properties of nanomaterials and the areas of negative differential resistance (NDR) leads to the creation of electronic components with predefined physical properties.

In this paper, the electric transport characteristics of the FeSe monolayer are modelled and analysed in the framework of the density functional theory in combination with the method of non-equilibrium Green's functions (DFT + NEGF). The first reason of our interest has been to simulate the electric transport properties of the FeSe monolayer, whose tetragonal structure of the crystal is very simple. It is completely characterised by two lattice parameters [23]. Second, when the temperature changes, FeSe undergoes small structural changes. This change between iron atoms causes the material to exhibit directionaldependent behaviour, such as the increased electrical resistance or conductivity, only in the left-to-right or forward-to-backward direction (nematic) [24].

The discovery of superconductivity in $2 \mathrm{D}-\mathrm{FeSe}$ at a critical temperature $\mathrm{T}_{\mathrm{C}} \sim 65 \mathrm{~K}$ was quite surprising, since in bulk $3 \mathrm{D}-\mathrm{FeSe}$ the $T_{C}$ value did not exceed $10 \mathrm{~K}$. A possible reason for this record-breaking increase in the critical temperature of the FeSe monolayer is a sharp increase in the electron concentration due to the presence of oxygen vacancies on the surface of the $\mathrm{SrTiO}_{3}$ substrate [25]. Note that paper [26] proposes an alternative approach to the deposition of potassium atoms on the surface of the FeSe monolayer to change the electron concentration. These features of the FeSe superconducting monolayer are insufficiently explained as part of the traditional phonon and spin-fluctuation mechanism of electron pairing, which requires the study of its electronic properties. 


\section{GEOMETRY}

The geometry of the FeSe model monolayer is shown in Fig. 1 (a-b). Exploration of electric transport characteristics was carried out with samples that were not-stressed (Fig. 1a), stressed with a substrate (Fig. 1b) and without it. When a monolayer is placed on a substrate, the structure is stressed and the linear dimensions increase.

The lattice constant in the layer of the non-stressed sample is $3.765 \AA$, and in the layer of a stressed sample it is significantly larger and is $3.905 \AA$. The length of the electrodes along the $\mathrm{C}$ axis of the nonstressed sample is $\sim 7.53 \AA$, and the stressed sample is $\sim 7.81 \AA$. The length of the monolayer under examination reaches $\sim 50.86 \AA$. The distance between the electrodes for a non-stressed and stressed structure is 35.8 $\AA$ and $37.14 \AA$, respectively.

a)
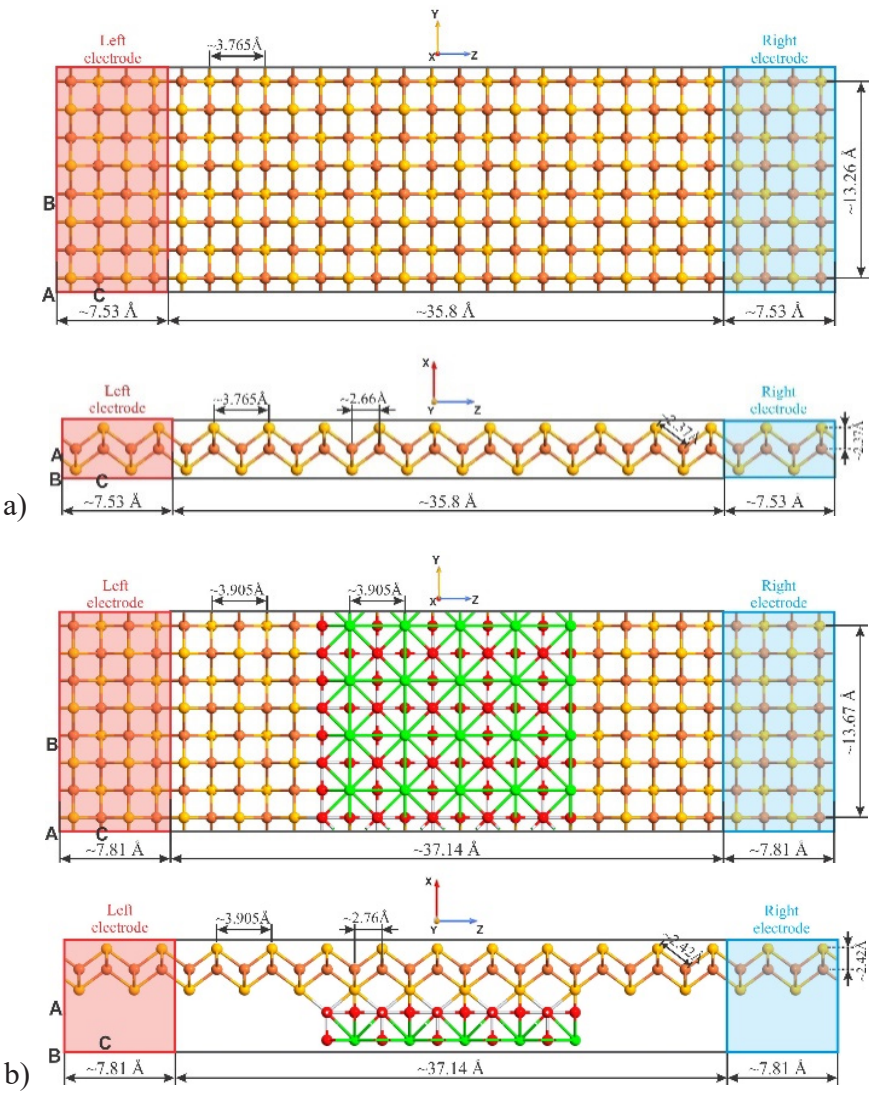

$\begin{array}{cccc}\text { Iron atom } & \text { Selenium atom } & \text { Strontium atom } \\ \begin{array}{c}\text { Titanium } \\ \text { atom }\end{array} & - & \text { Oxygen atom } & \end{array}$

Fig. 1. The geometry of the sample. 
For optimization, the 2D-FeSe geometry and description of the interaction between its atoms DFT were used with the exchange-correlation functional GGA-PBE [27], [28].

\section{SIMULATION MODEL AND METHODS}

Calculations of electric transport characteristics of 2D-FeSe were performed in the framework of DFT using the method of NEGF in the local density approximation (LDA) [29]. Modelling of quantum transport characteristics of a nanodevice is implemented in the Atomistix ToolKit with
Virtual NanoLab [30]. The main equations of this method are described in detail in our works [31], [32]. The 2D-FeSe CVC is calculated on the basis of the well-known Landauer equation, which indicates the fundamental relationship of the electric current with the transmission spectrum [33]:

$$
I\left(V_{L}, V_{R}, T_{L}, T_{R}\right)=\frac{2 e}{h} \int_{-\infty}^{+\infty} T(\varepsilon)\left[f\left(\frac{\varepsilon-\mu_{R}}{k_{B} T_{R}}\right)-f\left(\frac{\varepsilon-\mu_{L}}{k_{B} T_{L}}\right)\right] d \varepsilon
$$

where $e$ is the charge of the electron, $h$ is Planck's constant, $\varepsilon$ is energy, $T(\varepsilon)$ is function (spectrum) transmission, $f(\varepsilon)$ is the Fermi distribution function of quasiparticle energy, $k_{B}$ is the Boltzmann's constant, $T_{R}, T_{L}$ are the current temperatures and $\mu_{R}, \mu_{L}$ are the electrochemical potentials of the right and left electrode.

The differential conductivity of the FeSe monolayer was obtained from the selfconsistent current for a number of applied displacement and performing numerical differentiation.

\section{RESULTS AND DISCUSSION}

Evolution of the transmission spectrum, which increases with bias voltage of the monolayer from $-2 \mathrm{~V}$ to $2 \mathrm{~V}$, is shown in Fig. 2. The bias voltage increased in increments of $0.2 \mathrm{~V}$.

The transmission ability of the FeSe nanostructure increases gradually as the bias voltage grows. The structure of the spectrum gets complicated and new ones appear, preserving the old ones (Fig. 1a). Increase in the number of peaks gives information about increasing indication in the transport of quasiparticles through the structure under consideration [34]. At bias voltages from $0.4 \mathrm{~V}$ and higher (Fig. $2 \mathrm{a}$ ), peaks are observed at $-2.9 \mathrm{eV},-2.4 \mathrm{eV},-2.2$ $\mathrm{eV},-1.9 \mathrm{eV},-1.5 \mathrm{eV},-1.2 \mathrm{eV},-0.9 \mathrm{eV}, 0.9$ $\mathrm{eV}, 1.1 \mathrm{eV}, 1.2 \mathrm{eV}, 1.4 \mathrm{eV}, 1.6 \mathrm{eV}, 1.8 \mathrm{eV}$, $2 \mathrm{eV}, 2.3 \mathrm{eV}, 2.6 \mathrm{eV}, 2.9 \mathrm{eV}$. The maximum peak occurred at energies of $-1.2 \mathrm{eV}$ and $1.2 \mathrm{eV}$. A dip in the transmission spectrum was observed at about $0.1 \mathrm{eV}$. It should be noted that for values from $-2 \mathrm{~V}$ to $-1 \mathrm{~V}$, there is no transmission in the sample. From the transmission spectrum, we can conclude that resonant tunnelling is possible, which is explained by the low dimension of the FeSe monolayer. 


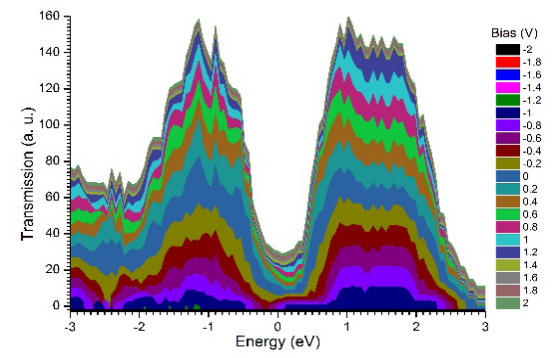

a)

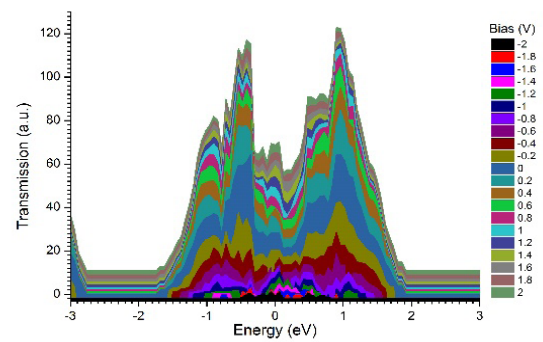

b)

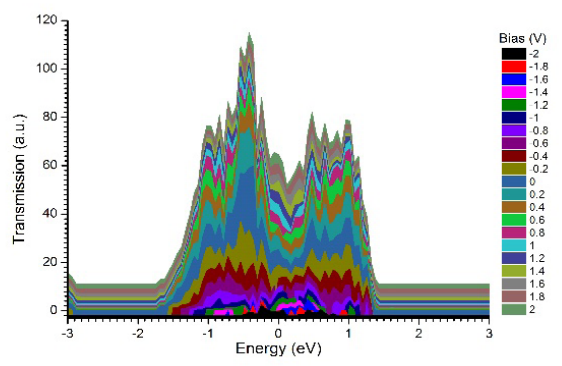

c)

Fig. 2. Evolution of the transmission spectra of a monolayer FeSe:

a) non-stressed sample;

b) stressed sample with a substrate;

c) stressed sample without a substrate.

There are fewer peaks in a stressed sample with a substrate (Fig. 2b), which indicates lower characteristics of the electronic transport of quasiparticles. In this case, the FeSe transmission is recorded from $-2 \mathrm{~V}$. The maximum transmission coefficient in this case is less than in a non-stressed sample. The electric transport characteristics are better in a sample with an increased order parameter and without a substrate, judging by the number of peaks, but the interval in which the maxima are observed is more than in a sample with a substrate.

The results of modelling the CVC and differential conductivity are shown in Fig. 3. (The CVC and differential conductivity of the nanostructures under consideration are calculated using Eqs. $(1,2))$.

On CVC of the nanostructure, it can be seen that the classical traditional parameter of order in the voltage ranges from $-2 \mathrm{~V}$ to $-1.2 \mathrm{~V}$, the dependence between current and voltage becomes linear and the current does not flow through the structure. A sharp drop was noted from $-1.2 \mathrm{~V}$ to $-1 \mathrm{~V}$. This is a section of NDR (Fig. 3a (1) and Fig. 3b (1)). The next section of the NDR is marked in the range from $1.2 \mathrm{~V}$ to $1.4 \mathrm{~V}$. The minimum was observed at $-0.8 \mathrm{~V}$. The maximum value of the non-stressed sample current was $30 \mu \mathrm{A}$. A section of almost linear dependence appeared in the range from 1.6 $\mathrm{V}$, where the current did not flow through the FeSe monolayer. On the curves 2 and 3 , negative differential resistance was not observed.

In the samples with an increased order parameter with and without $\mathrm{Sr}, \mathrm{Ti}, \mathrm{O}$ substrate, CVC was almost the same, differing only in the fact that a dip in the nanodevice 
with the substrate was at $-1.6 \mathrm{eV}$ and $\mathrm{CVC}$ became $\mathrm{N}$-shaped. The maximum current of $100 \mu \mathrm{A}$ was also recorded in the sample with the substrate. In both samples, the current increased in the range from $-1 \mathrm{~V}$ to $1 \mathrm{~V}$ from $-100 \mu \mathrm{A}$ to $80 \mu \mathrm{A}$.

In the differential conductivity spectrum in the range from $-2 \mathrm{~V}$ to $2 \mathrm{~V}$, we observed five peak structures of differential conductivity. Moreover, the intervals of NDR are characteristic only of a non-stressed film. In sample 1 (Fig. 3b) peaks were observed at $-1.3 \mathrm{~V},-0.7 \mathrm{~V}, 0 \mathrm{~V}, 1.1 \mathrm{~V}, 1.8 \mathrm{~V}$. In the sample, peak structures of $10 \mu \mathrm{S}, 70 \mu \mathrm{S}, 90$ $\mu \mathrm{S}, 290 \mu \mathrm{S}$ were recorded in the region of bias voltages of $-1.5 \mathrm{~V},-0.8 \mathrm{~V},-0.6 \mathrm{~V}, 0 \mathrm{~V}$,
$0.5 \mathrm{~V}, 0.9 \mathrm{~V}, 1.5 \mathrm{~V}$. There are a few maxima in sample 3, but they have higher differential conductivity. The maximum value of the differential conductivity in the negative side was $-230 \mu \mathrm{S}$, in the positive $290 \mu \mathrm{S}$.

Minima were observed around $-1.1 \mathrm{~V}$, $-1.7 \mathrm{~V}, 0.9 \mathrm{~V}, 1.3 \mathrm{~V}, 1.9 \mathrm{~V}$. The registration of negative differential resistance at both signs of the applied voltage indicates a symmetrical structure of the FeSe molecule [34]. The value of $\Delta V$ varied from $0.2 \mathrm{~V}$ to $0.7 \mathrm{~V}$. The nature of the resulting maxima on the differential conductivity spectrum is related to the Coulomb interaction of particles.

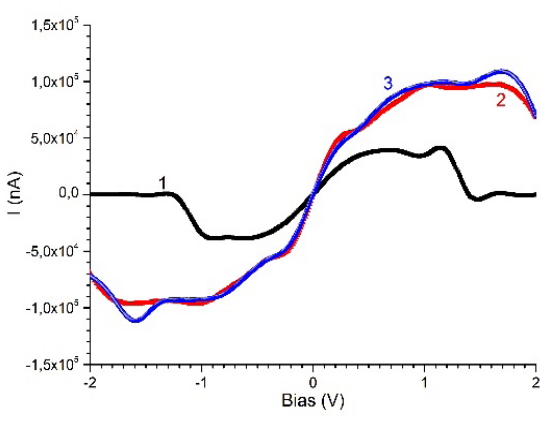

a)

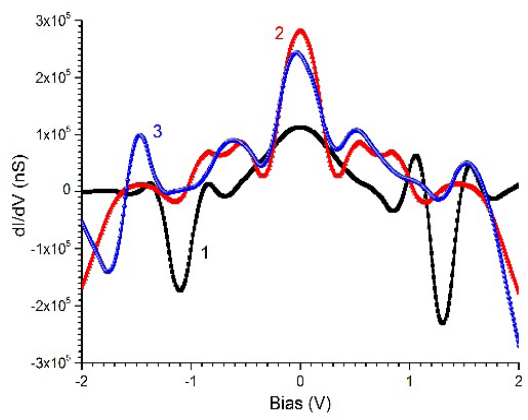

b)

Fig. 3. $I V$ (a) and $d I / d V$-characteristics (b) of the FeSe monolayer

( 1 - non-stressed sample, 2 - stressed sample with a substrate, 3 - stressed sample without a substrate).

The graph clearly shows that in a stressed monolayer without a substrate, electronic transport is faster, since the stress period in it is less than in a stressed sample with a substrate.

Figure 4 shows the dependence of the second derivative $\left(d^{2} I / d V_{2}\right)$ on the bias voltage. It characterises the features of electronphonon interaction in the FeSe monolayer with different geometric structures, the influence of substrates on the properties of the sample, and the nature of quasiparticle tunnelling.

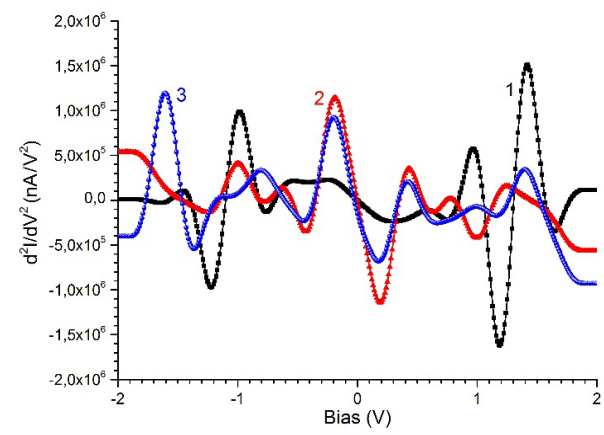

Fig. 4. $d^{2} I / d V^{2}$ - characteristics (b) of the FeSe monolayer ( 1 - non-stressed sample, 2 - stressed sample with a substrate, 3 - stressed sample without a substrate). 
Three distinct peaks were observed at $-1 \mathrm{~V}, 1 \mathrm{~V}$, and $1.5 \mathrm{~V}$ in a non-stressed sample (Fig. 4 (1)), relevant to the tunnelling conductivity of the FeSe sample. In the background structure of non-stressed sample, the background responsible for the electron-phonon interaction was observed at $-0.8 \mathrm{~V} \div 0.8 \mathrm{~V}$. The dips at -1.2 and $1.2 \mathrm{~V}$ (Fig. 4 (1)) are associated with phonon processes and can be compared with the peaks of the fluctuation spectrum of the structure. The most distinct peaks relevant to tunnelling occurred in a stressed sample without a substrate. The depth of the minimum at $0.2 \mathrm{~V}$ in a stressed sample with a substrate was $-12 \mu \mathrm{A} / \mathrm{V}^{2}$ (Fig. 3(2)) and in a stressed sample without a substrate it was $-0.6 \mu \mathrm{A} / \mathrm{V}^{2}$ (Fig. 3(3)). Thus, the local minimum was enhanced in the sample when exposed to the substrate in the region of 0.2 V. In stressed samples, the electron-phonon mechanism of tunnelling conductivity prevailed and there were more peaks in the region of $-1.8 \mathrm{~V},-1 \div 0.7 \mathrm{~V}, 0.2 \mathrm{~V}, 0.5 \mathrm{~V}, 1.2$ $\mathrm{V} \div 1.4 \mathrm{~V}$.

\section{CONCLUSIONS}

In this paper, the electric transport properties of the FeSe monolayer have been modelled in accordance with DFT + NEGF. Transmission spectra CVC, $d I / d V$, $d^{2} I / d V^{2}$ have been determined. It has been shown that the transmission of the nanostructure depends on the geometry of the sample and that the current flows well through the stressed film in contrast to the non-stressed one. The nature of sharp peaks in the transmission spectrum is related to resonant tunnelling of quasiparticles. On CVC of non-stressed sample, a section of NDR is observed in the range from $-1.2 \mathrm{~V}$ to -1 and from $1.2 \mathrm{~V}$ to $1.4 \mathrm{~V}$. NDR is shown at both signs of the applied voltage, which allows judging about the symmetry of the structure. Based on the second derivative of $\mathrm{CVC}$, we can conclude that the phonon conduction mechanism is manifested in the non-stressed sample. There are three distinct maxima and two minima. It has been shown that the electron-phonon interaction is stronger in a stressed FeSe film than in the non-stressed, and that the frequency of local fluctuation increases when exposed to the $\mathrm{SrTiO}_{3}$ substrate. The results obtained can be useful for the calculation of new promising electronic devices in $2 \mathrm{D}$ nanoelectronics.

\section{ACKNOWLEDGEMENTS}

The research has been supported by the grant of the Ministry of Education and Science of the Republic of Kazakhstan
AP08052562. In addition, the research of AIP has been supported by the LatvianUkrainian Grant LV-UA/2018/2.

\section{REFERENCES}

1. Kyeremateng, N.A., Brousse, T., \& Pech, D. (2017). Microsupercapacitors as Miniaturized Energy-Storage Components for On-
Chip Electronics. Nature Nanotechnology, 12, 7-15. DOI:10.1038/nnano.2016.196 
2. Kreisel, A., Hirschfeld, P.J., \& Andersen, B.M. (2020). On the Remarkable Superconductivity of $\mathrm{FeSe}$ and its Close Cousins. Symmetry, 12 (9), 1402. DOI:10.3390/sym12091402

3. Liu, C., \& Zou, K. (2020). Tuning Stoichiometry and its Impact on Superconductivity of Monolayer and Multilayer FeSe on $\mathrm{SrTiO}_{3}$. Physical Review B, 101 (14), 140502. DOI:10.1103/ PhysRevB.101.140502

4. Chen, Y.H., Sun, Y., Ji, S.Y., Xiong, W., Pei, Z.C., \& Wang, Z.W. (2020). Enhancement of Effective Masses of the Surface Polaron in FeSe Thin Film on $\mathrm{SrTiO}_{3}$ Substrate. Superlattices and Microstructures, 106573. DOI:10.1016/j.spmi.2020.106573

5. Kozlovskiy, A., Zhanbotin, A., Zdorovets, M., Manakova, I., Ozernoy,A., Kadyrzhanov, K., \& Rusakov, V. (2015). Study of Ni/Fe Nanotube Properties. Nuclear Instruments and Methods in Physics Research Section B: Beam Interactions with Materials and Atoms, 365, 663-667. DOI:10.1016/j. nimb.2015.09.090

6. Zdorovets, M.V., \& Kozlovskiy, A.L. (2018). Argon Ion Irradiation Effect on Zn Nanotubes. Journal of Materials Science: Materials in Electronics, 29 (5), 3621-3630. DOI:10.1007/s10854-017-8292-5

7. Zdorovets, M.V., \& Kozlovskiy, A.L. (2019). Investigation of Phase Transformations and Corrosion Resistance in $\mathrm{Co} / \mathrm{CoCo}_{2} \mathrm{O}_{4}$ Nanowires and their Potential Use as a Basis for Lithium-Ion Batteries. Scientific Reports, 9 (1), 1-12. DOI:10.1038/s41598019-53368-y

8. Akilbekov, A., Akylbekova, A., Usseinov, A., Kozlovskyi, A., Baymukhanov, Z., Giniyatova, S., ... \& Dauletbekova, A. (2020). Ion Track Template Technique for Fabrication of $\mathrm{ZnSe}_{2} \mathrm{O}_{5}$ Nanocrystals. Nuclear Instruments and Methods in Physics Research Section B: Beam Interactions with Materials and Atoms, 476, 10-13. DOI:10.1016/j.nimb.2020.04.039

9. Chen, C., Liu, C., Liu, Y., \& Wang, J. (2020). Bosonic Mode and ImpurityScattering in Monolayer Fe(Te,Se) HighTemperature Superconductors. Nano
Letters, 20 (3), 2056-2061. DOI:10.1021/ acs.nanolett.0c00028

10. Isherwood, L.H., Worsley, R.E., Casiraghi, C., \& Baidak, A. (2018). Alpha Particle Irradiation of Bulk and Exfoliated $\mathrm{MoS}_{2}$ and $\mathrm{WS}_{2}$ Membranes. Nuclear Instruments and Methods in Physics Research Section B: Beam Interactions with Materials and Atoms, 435, 180-189. DOI:10.1016/j. nimb.2018.01.018

11. Qiao, M., Wang, T.J., Zhang, J., Liu, Y., Liu, P., \& Wang, X.L. (2018). The Effect of Carbon-Ion Irradiation on Surface Microstructure and Photoluminescence Properties in Monolayer Tungsten Diselenide. Nuclear Instruments and Methods in Physics Research Section B: Beam Interactions with Materials and Atoms, 435, 278-284. DOI:10.1016/j. nimb.2018.01.003

12. Sergeyev, D.M. (2018). Computer Simulation of Electrical Characteristics of a Graphene Cluster with Stone-Wales Defects. Journal of Nano and Electronic Physics, 10 (3), 03018. DOI:10.21272/ jnep.10(3).03018

13. Dragoman, M.A., Dinescu, A., \& Dragoman, D. (2019). 2D Materials Nanoelectronics: New Concepts, Fabrication, Characterization from Microwaves up to Optical Spectrum. Phys. Status Solidi A, 1800724. DOI:10.1002/pssa.201800724

14. Illarionov, Yu.Yu., Knobloch, T., Jech, M., Lanza, M., Akinwande, D., Vexler, M.I., ... \& Grasser, T. (2020). Insulators for 2D Nanoelectronics: the Gap to Bridge. Nature Communications, 11, 3385. DOI:10.1038/ s41467-020-16640-8

15. Sergeyev, D., \& Zhanturina, N. (2019). Simulation of Electrical Characteristics of Switching Nanostructures "Pt-TiOPt" and "Pt-NiO-Pt" with Memory. Radioengineering, 28 (4), 714-720. DOI:10.13164/re.2019.0714

16. Liu, N., Bo, G., Liu,Y., Xu, X., Du, Y., \& Dou, Sh. (2019). Recent Progress on Germanene and Functionalized Germanene: Preparation, Characterizations, Applications, and Challenges. Small, 15(32), 1805147. DOI:10.1002/smll.201805147 
17. Huang, W.Q., Liu, Sh.R., Pen, H.Y., Li, X., \& Huang, Z.M. (2020). Synthesis of New Silicene Structure and its Energy Band Properties. Chinese Physics B, 29, 084202. DOI:10.1088/1674-1056/ab942c

18. Kiraly, B., Liu, X., Wang, L., Zhang, Zh., Mannix, A.J., Fisher, B.L., ... \& Guisinger, N.P. (2019). Borophene Synthesis on $\mathrm{Au}(111)$. ACS Nano, 13 (4), 3816-3822. DOI:10.1021/acsnano.8b09339

19. Sahoo, S.K., \& Wei, K.H. (2019). A Perspective on Recent Advances in 2D Stanene Nanosheets. Advanced Materials Interfaces, 6 (18), 1900752. DOI:10.1002/ admi.201900752

20. Pica,M.,\&D'Amato, R.(2020). Chemistry of Phosphorene: Synthesis, Functionalization and Biomedical Applications in an Update Review. Inorganics, 8 (4), 29. DOI:10.3390/ inorganics8040029

21. Vergera, L., Xub, Ch., Natua, V., Cheng, H., Ren, W., \& Barsouma, M.W. (2019). Overview of the Synthesis of MXenes and Other Ultrathin 2d Transition Metal Carbides and Nitrides. Current Opinions in Solid State and Materials Science, 23 (3), 149163. DOI:10.1016/j.cossms.2019.02.001

22. Meng, Zh., Stolz, R.M., Mendecki, L., \& Mirica, K.A. (2019). ElectricallyTransduced Chemical Sensors Based on Two-Dimensional Nanomaterials. Chemical Reviews, 119, 478-598. DOI:10.1021/acs. chemrev.8b00311

23. Yu, J., Meng, L., Wu, J., \& Li, Y. (2019). Correlation Effect on the Electronic Properties of Pair-Checkerboard AFM Monolayer FeSe: a First-Principles Study. Journal of Phyics: Condensed Matter, 31, 305502. DOI:10.1088/1361-648X/ab1afb

24. Koch, R.J., Konstantinova, T., Abeykoon, M., Wang, A., Petrovic, C., Zhu, Y., ... \& Billinge, L. (2019). Room Temperature Local Nematicity in FeSe Superconductor. Physical Review B 100, 020501. DOI:10.1103/PhysRevB.100.020501

25. Jandke, J. Yang, F., Hlobil, P., Engelhardt, T., Rau, D., Zakeri, K., ... \& Wulfhekel, W. (2019). Unconventional Pairing in Single FeSe Layers. Physical Review B 100, 020503(R). DOI:10.1103/
PhysRevB.100.020503

26. Coh, S., Cohen, M.L., \& Louie, S.G. (2015). Large Electron-Phonon Interactions from FeSe Phonons in a Monolayer. New Journal of Physics, 17, 073027. DOI:10.1088/13672630/17/7/073027.

27. Sergeyev, D. (2020) Single Electron Transistor Based on Endohedral Metallofullerenes $\mathrm{Me} @ \mathrm{C}_{60} \quad(\mathrm{Me}=\mathrm{Li}$, $\mathrm{Na}, \mathrm{K})$. Journal of Nano and Electronic Physics, 12 (3), 03017. DOI:10.21272/ jnep.12(3).03017

28. Sergeyev, D.M. (2020). Specific Features of Electron Transport in a Molecular Nanodevice Containing a Nitroamine Redox Center, Technical Physics, 15 (4), 573-577. DOI:10.1134/S1063784220040180

29. Sergeyev, D.M., Myasnikova, L.N., \& Shunkeyev, K.Sh. (2020). Computer Simulation of Spin Filtration Properties of Zigzag-Edged Octagraphene Nanoribbon Saturated with Hydrogen Atoms. Russian Physics Journal, 63, 303-310. DOI:10.1007/ s11182-020-02036-0

30. Atomistix ToolKit. Manual Version. (2015). QuantumWise A/S, 1, 840.

31. Ryczko, K., Strubbe, D.A., \& Tamblyn, I. (2019). Deep Learning and DensityFunctional Theory. Physical Review A, 100, 022512. DOI: 10.1103/ PhysRevA.100.022512

32. Sergeyev, D. (2019). Computer Simulation of the Electrotransport Characteristics of the " $\mathrm{Au}$ - Bipyridine - Au" Nanocontact. Journal of Nano and Electronic Physics, 11 (4), 04023 DOI:10.21272/jnep.11(4).04023

33. Gurvitz, S. (2019). Generalized Landauer Formula for Time-Dependent Potentials and Noise-Induced Zero-Bias DC Current. Journal of Physics A: Mathematical and Theoretical, 52,175301.DOI:10.1088/17518121/ab10ed

34. Esmaeili, M., Jafari, M., \& Sanaeepur, M. (2020). Negative Differential Resistance in Nanoscale Heterostructures Based on Zigzag Graphene Nanoribbons AntiSymmetrically Decorated with BN. Superlattices Microstructures, 145, 106584. DOI:10.1016/j.spmi.2020.106584 